\title{
Intermittent Transport across the Scrape-off Layer: Latest Results from ASDEX Upgrade
}

\author{
M Kočan ${ }^{1, *}$, H W Müller ${ }^{1}$ ， B Nold ${ }^{2}$, T Lunt ${ }^{1}$, J Adámek ${ }^{3}$, S Y Allan ${ }^{4}$, \\ M Bernert ${ }^{1}$, G D Conway ${ }^{1}$, P de Marné $^{1}$, T Eich $^{1}$, S Elmore ${ }^{4}$, \\ F P Gennrich $^{5}$, A Herrmann ${ }^{1}$, J Horacek ${ }^{3}$, Z Huang ${ }^{2}$, A Kallenbach ${ }^{1}$, \\ M Komm ${ }^{3}$, M Maraschek ${ }^{1}$, F Mehlmann ${ }^{5}$, S Müller ${ }^{1}$, T T Ribeiro ${ }^{1}$, \\ V Rohde ${ }^{1}$, R Schrittwieser ${ }^{5}$, B Scott ${ }^{1}$, U Stroth ${ }^{1}$, W Suttrop ${ }^{1}$, E Wolfrum ${ }^{1}$ \\ and the ASDEX Upgrade Team
}

${ }^{1}$ Max-Planck-Institut für Plasmaphysik, EURATOM Association, 85748 Garching, Germany

${ }^{2}$ Institut für Plasmaforschung, Universität Stuttgart, 70569 Stuttgart, Germany

${ }^{3}$ Association EURATOM-IPP.CR, Za Slovankou 3, 18200 Prague 8, Czech Republic

${ }^{4}$ EURATOM/CCFE Fusion Association, Culham Science Centre, Abingdon, Oxon, OX14 3DB, UK

${ }^{5}$ Institute for Ion Physics and Applied Physics, Association Euratom-ÖAW, Austria

* Present address: ITER Organization, Route de Vinon sur Verdon, 13115 Saint Paul Lez Durance, France

E-mail contact of main author: martin.kocan@iter.org

\begin{abstract}
.
We report the latest results of turbulence and transport studies in the ASDEX Upgrade scrapeoff layer (SOL). Dissimilarity between the plasma and the floating potential fluctuations is studied experimentally and by gyrofluid simulations. Measurements by a retarding field analyzer reveal that both, ELM and turbulent filaments convey hot ions over large radial distances in the SOL. The measured far SOL ELM ion temperature increases with the ELM energy, consistent with earlier observations that large ELMs deposit a large fraction of their energy outside the divertor. In the SOL, the ELM suppression by magnetic perturbations (MPs) results into lower ELM ion energy in the far SOL. At the same time, large filaments of ion saturation current are replaced by more continuous bursts. Splitting of the divertor strike zones observed by the infrared imaging in H-mode with MPs agree with predictions from the EMC3Eirene simulations. This suggests that the 'lobe' structures due to perturbation fields observed near the X-point are not significantly affected by plasma screening, and can be described by a vacuum approach, as in the EMC3-Eirene. Finally, some effects of the MPs on the L-mode SOL are addressed.
\end{abstract}

Keywords: ASDEX Upgrade, scrape-off layer, turbulence, edge localized modes, magnetic perturbations

PACS: 52.55.Fa, 52.25.Fi, 52.65.Tt, 52.70.Ds, 52.70.Kz 


\section{Introduction}

Convective transport of hot and dense plasma filaments ${ }^{1}$ in the scrape-off layer (SOL) due to ELMs and turbulence will place wear on plasma facing components (PFCs) and affect the dust production and the tritium inventory in burning plasma reactors. Getting as much information as possible from today's tokamaks is essential for understanding of the heat and particle transport in the SOL, which is critical for predicting the plasma-wall interactions in ITER and beyond. This contribution highlights the latest research of intermittent transport in the SOL of the ASDEX Upgrade (AUG) tokamak. Emphasis is given to electric probe measurements. The key diagnostic systems for turbulence and transport studies in the AUG SOL are briefly described in section 2. Individual research topics are covered in the following sections: 3 - L-mode and inter-ELM turbulence, 4 - Far SOL ELM ion energies, 5 Influence of magnetic perturbations on SOL transport. Summary is given in section 6.

\section{Diagnostic set up}

In AUG, as in any tokamak, turbulence and transport in the SOL is studied mainly by electric probes. The probe system in AUG is depicted in figure 1. The workhorse diagnostic is a horizontal reciprocating probe manipulator (RPM) located $31 \mathrm{~cm}$ above the outboard midplane. The RPM is used to immerse various advanced electric probes into the plasma ([1] and Table 1). Two other reciprocating probe systems - a Langmuir probe (LP) with radially separated pins for filament transport studies (dubbed 'filament probe', FP) [2] and a new retarding field analyzer, dubbed 'RFA2' (another identical RFA can be mounted on RPM) are installed inside the torus on magnetically-driven manipulators. Probe data are measured at an acquisition frequency of $2 \mathrm{MHz}$ with 14-bit ADC resolution. During the reciprocation, the probes can be maintained for a programmed time interval at fixed outboard midplane separatrix distance, $\Delta \mathrm{r}_{\text {sep }}$. As illustrated in figure 1 , the power fluxes estimated from probe measurements can be compared with observations from an infrared (IR) camera (framing frequency up to $25 \mathrm{kHz}$ ) viewing the RPM and the FP. The measurements by probes in the main SOL are complemented with data from other pertinent diagnostics such as e.g. flushmounted Langmuir probes [3], X-point reciprocating probe [4], divertor thermography, visible light imaging and the multi-channel Doppler reflectometers [5].

\section{L-mode and inter-ELM turbulence}

Cross-field turbulent transport during inter-ELM periods will be one of the major causes of erosion of the beryllium first wall in ITER [6]. An insight into turbulent processes is traditionally gained by studying L-mode SOL which bears a lot of resemblance to inter-ELM SOL but the absence of ELMs makes it less harsh for probe measurements.

\subsection{The importance and feasibility of direct plasma potential measurements}

The fluctuation-induced radial particle flux, $\Gamma_{\mathrm{r}}$ is among the most important parameters characterizing turbulent transport. Ideally, $\Gamma_{\mathrm{r}}$ should be evaluated from the fluctuations of the plasma density and the plasma potential as $\Gamma_{\mathrm{r}} \propto\left\langle\tilde{\mathrm{n}}_{\mathrm{e}} \nabla_{\theta} \tilde{\mathrm{V}}_{\mathrm{p}}\right\rangle$. Since the measurements of $\tilde{\mathrm{n}}_{\mathrm{e}}$ and $\tilde{\mathrm{V}}_{\mathrm{p}}$ are beyond the capability of a simple Langmuir probe, $\Gamma_{\mathrm{r}}$ is almost never derived in this way. Instead, $\Gamma_{\mathrm{r}}$ is estimated from the most easily measurable fluctuations of ion saturation current $\left(\tilde{\mathrm{I}}_{\text {sat }}\right.$ ) and floating potential $\left(\tilde{\mathrm{V}}_{\mathrm{f}}\right.$ ) by assuming $\tilde{\mathrm{n}}_{\mathrm{e}} \propto \tilde{\mathrm{I}}_{\text {sat }}$ and $\tilde{\mathrm{V}}_{\mathrm{p}} \approx \tilde{\mathrm{V}}_{\mathrm{f}}$. In AUG, the legitimacy of this assumption was tested in an experiment in which $\tilde{\mathrm{n}}_{\mathrm{e}}, \tilde{\mathrm{V}}_{\mathrm{p}}, \tilde{\mathrm{I}}_{\mathrm{sat}}, \tilde{\mathrm{V}}_{\mathrm{f}}$ and $\tilde{\mathrm{T}}_{\mathrm{e}}$

\footnotetext{
${ }^{1}$ The term 'filament' is typically used for field-aligned structures observed during ELMs, while the turbulent coherently propagating objects are more often called 'blobs', referring of their cross-field appearance. Since both, blobs and ELM filaments, are field-aligned objects evolving in the SOL in the same manner, in this paper we refer to both ELM and turbulence structures simply as filaments.
} 
(with $\widetilde{\mathrm{T}}_{\mathrm{e}}$ the electron temperature fluctuations) were measured simultaneously near the separatrix from conditionally-sampled probe current-voltage (I-V) characteristics and from an emissive probe (EP) [7]. These probe collectors were a part of a single probe head (dubbed 'turbulence probe') mounted on the RPM. The main result of this experiment is illustrated in figure 2. It shows that $\tilde{\mathrm{V}}_{\mathrm{f}}$ and $\tilde{\mathrm{V}}_{\mathrm{p}}$ are anti-correlated due to $\tilde{\mathrm{T}}_{\mathrm{e}}$ (a strong influence of electron temperature on plasma potential measurements was observed also in earlier experiments in AUG and elsewhere [1]). This observation indicates that, at least near the separatrix, the assumption $\tilde{\mathrm{V}}_{\mathrm{p}} \approx \tilde{\mathrm{V}}_{\mathrm{f}}$ does not conform to experimental observations and cannot yield correct estimates of $\Gamma_{\mathrm{r}}$ other than by coincidence. It is also worth noticing that this observation is consistent with the accompanying simulations of probe measurements in a turbulent SOL [7, 8] using the 3-d electromagnetic gyrofluid code GEMR [9]. As an illustrative example, $\Gamma_{\mathrm{r}}$ derived from synthetic probe measurements of $\tilde{\mathrm{I}}_{\mathrm{s}}$ and $\tilde{\mathrm{V}}_{\mathrm{f}}$ is compared in figure 3 with $\Gamma_{\mathrm{r}}$ obtained directly from $\tilde{\mathrm{n}}_{\mathrm{e}}$ and $\tilde{\mathrm{V}}_{\mathrm{p}}$. The former yields erroneously large $\Gamma_{\mathrm{r}}$, which could be avoided in experiment by measuring the plasma potential. A recent experiment in AUG, in which $\mathrm{V}_{\mathrm{p}}$ was assessed by two independent techniques, demonstrates the feasibility of such measurements. In this experiment, which will be presented in a future paper, the RPM was equipped with a probe head consisting of a ball pen probe (BPP) which measures a potential close to $V_{p}[10,11]$, and an electrically floating LP. As shown in figure 4, at the turning point of the reciprocation, the LP reaches the temperature at which it undergoes a transition to an $\mathrm{EP}$, providing additional measurements of $\mathrm{V}_{\mathrm{EP}} \approx \mathrm{V}_{\mathrm{p}}$ during the probe outward motion. The high frequency components of the potential fluctuations from the LP (most likely due to $T_{e}$ fluctuations) are strongly reduced after the transition to the EP and the frequency spectrum becomes similar to that of the BPP. Observing that two techniques measure similar values of $\mathrm{V}_{\mathrm{p}}$ is encouraging. We also recall a good agreement of $\mathrm{E}_{\mathrm{r}} \approx \nabla \mathrm{V}_{\mathrm{p}}$ from the BPP with $\mathrm{E}_{\mathrm{r}}$ from Doppler reflectometry in AUG [1]. Now, when the diagnostics for $\mathrm{V}_{\mathrm{p}}$ measurements are becoming mature enough, it will be important to verify if the strong difference in amplitude and fluctuation phase of $V_{p}$ and $V_{f}$, observed in Ref. [7] for particular plasma conditions, generally applies to a SOL plasma.

\subsection{Ion energies in turbulent plasma filaments}

Ion temperature in turbulent plasma filaments $T_{i, f i l}$, affects the filament dynamics in several ways [12]. However, because of the complexity of modeling finite ion temperature effects, most turbulent models assume the cold ion approximation, i.e. $\mathrm{T}_{\mathrm{i} \text {,fil }}<<\mathrm{T}_{\mathrm{e} \text {,fil }}$, with $\mathrm{T}_{\mathrm{e} \text {,fil }}$ the filament electron temperature. It is unrealistic to expect that this assumption holds in the tokamak SOL where less mobile ions are naturally hotter than electrons [13]. Some recent modeling work addressed the effect of finite $T_{i, \text { fil }}[14,15]$ and have shown that, for example, the growth rate of linear drift waves decreases moderately and the convective rate of filaments increases as $T_{i, f i l}$ becomes larger than $T_{e, f i l}$. Further progress in addressing finite ion temperature effects is partly impeded by a lack of $\mathrm{T}_{\mathrm{i}, \mathrm{fil}}$ measurements.

First measurements of $T_{i, f i l}$ were recently obtained in AUG by an RFA mounted on the RPM. These measurements indicate that turbulent filaments convey hot ions over large radial distances in the SOL and provide evidence that, in contrast to the cold-ion assumption, $\mathrm{T}_{\mathrm{i}, \text { fil }}$ can be substantially larger than $\mathrm{T}_{\mathrm{e}, \text { fil }}$.

As depicted in figure 5, an RFA uses a series of grids shielded behind a narrow slit. One of the grids is swept positively to remove ions with energy below the grid potential, $\mathrm{V}_{\mathrm{g}}$. Another grid (labeled as 'grid 2') is used to suppress the secondary electrons emitted inside the analyzer. A collector measures the current of ions $\left(I_{c}\right)$ with energy above $\mathrm{eV}_{\mathrm{g} 1}$. The information about $\mathrm{T}_{\mathrm{i} \text {,fil }}$ was extracted using a conditional sampling technique from Ref. [16] 
(the same method was recently used to infer $T_{i, \text { fil }}$ from the ion sensitive probe characteristics measured in the linear device NAGDIS-II [17]). Filaments characterized by a similar ion current density, $\mathrm{j}_{\text {sat,fil }}$, monitored by the negatively-biased RFA slit plate, were selected from the measured time trace. As illustrated in figure $6, \mathrm{~T}_{\mathrm{i}, \text { fil }}$ was obtained from the e-folding voltage of the corresponding $I_{c}$ plotted against $V_{g 1}$, measured in ohmic discharges. The filament ion temperature from figure 6 tends to decrease radially with the decay length of $\lambda_{\mathrm{Ti}}$ $\approx \Delta \mathrm{r} / \log \left[\mathrm{T}_{\mathrm{i}, \mathrm{fil}}\left(\mathrm{r}_{1}\right) / \mathrm{T}_{\mathrm{i}, \text { fil }}\left(\mathrm{r}_{2}\right)\right] \approx 2 / \log (47 / 16) \approx 2 \mathrm{~cm}$. Additionally, figure 7 indicates that more intense filaments are characterized by somewhat higher $\mathrm{T}_{\mathrm{i}, \text { fil }}$. At the midplane separatrix distance $\Delta \mathrm{r}_{\text {sep }}=21 \mathrm{~mm}, \mathrm{~T}_{\mathrm{i}, \text { fil }}$ is up to $70 \%$ of the ion temperature at the separatrix, $\mathrm{T}_{\mathrm{i}, \mathrm{sep}}$, obtained from spectroscopic measurements. A factor of 2 larger $\mathrm{T}_{\mathrm{i}, \mathrm{fil}}$ at $\Delta \mathrm{r}_{\text {sep }}=21 \mathrm{~mm}$ from figure 7 compared to $\mathrm{T}_{\mathrm{i}, \text { fil }}$ at $\Delta \mathrm{r}_{\text {sep }}=25 \mathrm{~mm}$ from figure 6 can be partly explained by $\sim 30 \%$ longer parallel magnetic connection length $\mathrm{L}_{/ /}$in the plasma pulse from figure 7 , and thus weaker parallel loss $\propto \mathrm{c}_{\mathrm{s}} / \mathrm{L}_{/ /}$(with $\mathrm{c}_{\mathrm{s}}$ the ion sound speed). Assuming that turbulent filaments originate near the separatrix [18] (i.e. $\mathrm{T}_{\mathrm{i}, \mathrm{fil}}=\mathrm{T}_{\mathrm{i}, \mathrm{sep}}$ at the filament birth location), $\lambda_{\mathrm{Ti}} \approx$ $\Delta \mathrm{r}_{\text {sep }} / \log \left(\mathrm{T}_{\mathrm{i}, \text { sep }} / \mathrm{T}_{\mathrm{i}, \mathrm{fil}}\right) \approx 3-7 \mathrm{~cm}$ can be estimated from the measurements shown in figure 7 . This value of $\lambda_{\mathrm{Ti}}$ can be compared with $\lambda_{\mathrm{Ti}} \approx 2 \mathrm{~cm}$ obtained from the data from figure 6 , measured at $\Delta \mathrm{r}_{\text {sep }}=25-45 \mathrm{~mm}$. The observation that $\mathrm{T}_{\mathrm{i} \text {,fil }}$ drops faster radially further away from the separatrix suggests that the filament radial propagation velocity, $\mathrm{v}_{\mathrm{rad}} \approx \lambda_{\mathrm{Ti}} \mathrm{c}_{\mathrm{s}} / \mathrm{L}_{/ /}$, decreases as the filaments rarify due to parallel loss. The reliability of the conditional-sampling technique used to obtain $\mathrm{T}_{\mathrm{i} \text {,fil }}$ was tested on artificial RFA measurements generated by the GEMR simulations [19, 20]. These simulations addressed various aspects of ion temperature measurements in turbulent SOL by an RFA. Such instrumental study can be particularly important now, when RFAs are being installed in a number of tokamaks.

The measured $T_{i, f i l}$ was used to estimate $v_{\text {rad }}$ from a fluid model of the parallel filament transport in the SOL, described in Ref. [21]. Despite of its relative simplicity, the model was previously successful in reproducing a variety of experimental observations in AUG and JET [19, 21-25]. Additionally, in Ref. [24] the results obtained from the fluid model were consistent with the Monte Carlo simulations of ELM filaments [26]. In the present simulations, $\mathrm{v}_{\mathrm{rad}}$ was adjusted to match the measured $\mathrm{T}_{\mathrm{i}, \text { fil }}$. As shown in figure 7 , the required $\mathrm{v}_{\mathrm{rad}}$ is in the range of $400-1000 \mathrm{~m} \mathrm{~s}^{-1}$, which conforms to earlier measurements of $\mathrm{v}_{\mathrm{rad}}$ [12]. A tendency for $\mathrm{v}_{\mathrm{rad}}$ to increase with the filament particle density, as observed in figure 7 , is consistent with the interchange model of the filament dynamics in the SOL [27] which predict that denser filaments are subject to faster radial advection. RFA measurements also revealed some similarities of L-mode and inter-ELM turbulent transport in the SOL. These new measurements of inter-ELM transport in the AUG far SOL were reported in Ref. [28].

Following the technique originally introduced by Wan et al. [29], in some discharges the bias scheme of the RFA electrodes was periodically changed between the 'ion' and 'electron' mode (indicating the charges of which parallel energy distribution is measured) in order to measure both, $\mathrm{T}_{\mathrm{i}, \text { fil }}$ and $\mathrm{T}_{\mathrm{e}, \mathrm{fil}}$, during a single reciprocation. The bias voltages and the measured currents are shown in figure 5. The ion mode was described above. In the electron mode, both, ions and electrons, are allowed to enter the analyzer. Ions are repelled from the collector by a high positive voltage applied to grid 1. Grid 2 is swept negatively and the collector measures the current of electrons with the energy above $\mathrm{eV}_{\mathrm{g} 2}$. As can be seen in figure 5, the currents measured in the electron mode feature the same filamentary structure as in the ion mode. Using the aforementioned conditional sampling technique, the filament electron I-V characteristic was obtained from the data measured at $\Delta \mathrm{r}_{\text {sep }}=25 \mathrm{~mm}$ and is plotted in figure 6 . As can be seen from figure 5, in the electron mode, there is a positive offset of the collector current, $\mathrm{I}_{\mathrm{off}}$, when both, $\mathrm{V}_{\mathrm{g} 1}$ and $\mathrm{V}_{\mathrm{g} 2}$, are biased to high voltages so that neither ions nor electrons should reach the collector. For the moment the origin of $\mathrm{I}_{\mathrm{off}}$ is unknown, and, $\mathrm{I}_{\text {off }}$ is subtracted from $I_{c}$ plotted in figure 6 . An exponential fit to the slope of the $\mathrm{I}-\mathrm{V}$ characteristic 
gives $\mathrm{T}_{\mathrm{e}, \mathrm{fil}} \approx 12 \mathrm{eV}$. This can be compared with $\mathrm{T}_{\mathrm{i}, \text { fil }} \approx 47 \mathrm{eV}$ from figure 6 (left), measured during the same reciprocation in the ion mode. The observation $T_{i, f i l}>T_{e, f i l}$ is consistent with faster parallel cooling of more mobile electrons. It is worth mentioning that $\mathrm{T}_{\mathrm{e}, \mathrm{fil}}$ measured by the RFA is consistent with $\mathrm{T}_{\mathrm{e}, \mathrm{fil}} \approx 19 \mathrm{eV}$ at $\Delta \mathrm{r}_{\text {sep }}=14-18 \mathrm{~mm}$ measured earlier in AUG from the conditionally sampled Langmuir probe I-V characteristics, albeit in a different L-mode discharge [7].

Furthermore, two new electric probes were being designed for the RPM in AUG in the collaboration with the Institute of Plasma Physics in Prague, charting separate paths in SOL $T_{i}$ measurements. The first probe $-\mathrm{a}$ ball pen probe - measures $\mathrm{T}_{\mathrm{i}}$ by virtue of a positively swept collector recessed below the probe leading edge, similar to the Katsumata probe [30]. In AUG, the BPP was operated with fast voltage sweeping frequencies, aiming to measure $T_{i}$ fluctuations [31]. The second probe - an $\mathbf{E} \times \mathbf{B}$ analyser - is similar to an analyzer used in the DITE tokamak [32]. The $\mathbf{E} \times \mathbf{B}$ analyzer, recently used in the first experiment in AUG, is dedicated for measurements of $\mathrm{T}_{\mathrm{i}}$ fluctuations in the SOL. As shown in figure 8, in an $\mathbf{E} \times \mathbf{B}$ analyzer the ions are transmitted through a narrow aperture and enter the region of uniform electric field $\mathbf{E}$, generated by applying constant voltages to a pair of planar electrodes. The resulting $\mathbf{E} \times \mathbf{B}$ drift disperses the ions along an array of collectors. The spatial distribution of ions along the collectors is a function of the parallel ion velocity, $\mathrm{v}_{/}$, so that the parallel ion velocity distribution at the probe surface, $\mathrm{f}_{\mathrm{i}}\left(\mathrm{v}_{/ /}\right)$, as well as $\mathrm{T}_{\mathrm{i}}$ can be inferred from the collector currents (recently, a least-squares regularization method was used to extract $\mathrm{f}_{\mathrm{i}}\left(\mathrm{v}_{/ /}\right)$ from the time-averaged data measured by the RFA in the AUG L-mode plasma [33]).

\section{Far SOL ELM ion energies}

Experiments in the past few years have shown that ELMs can reach non-divertor PFCs with a large fraction of their initial energy [13, 21-25, 34, 35]. In ITER, the ELM-wall interactions can produce impurities, which are more likely to contaminate the confined plasma compared with those originating from the divertor. For fuel ions and low-Z impurities, the intensity of ELM-wall interactions is determined by ELM ion energies in the far SOL. However, with exception of earlier measurements on JET [22], AUG [24, 35] and MAST [36], far SOL ELM ion energies were practically unknown, making predictions towards ITER uncertain.

Systematic measurements of the far SOL ELM ion energies have been performed in AUG using the RFA mounted on the RPM. Illustrative results discussed here appear in more detail in Ref. [25]. Figure 9 shows typical time traces of $j_{\text {sat }}$ and $I_{c}$ measured by the RFA in two similar type I ELMs at $\Delta \mathrm{r}_{\text {sep }}=35 \mathrm{~mm}$. Note that both, $\mathrm{j}_{\mathrm{sat}}$ and $\mathrm{I}_{\mathrm{c}}$, feature a rich filamentary structure observed from earlier LP and IR measurements (see references in Ref. [25]). The ELM filamentary structure measured by the RFA was also found to be well correlated with that observed simultaneously by the visible light imaging [25]. Figure 9 shows that $I_{c}$ drops with increasing $\mathrm{V}_{\mathrm{g} 1}$ due to reflection of low energy ELM ions by the RFA grid. The characteristic far SOL ELM ion energy can be estimated from the collector current e-folding voltage. This is a principle of the conditional sampling method used in [25], which yields the ELM-averaged ion temperature, $\mathrm{T}_{\mathrm{i}, \mathrm{ELM}}$, shown in figure $9 . \mathrm{T}_{\mathrm{i}, \mathrm{ELM}}$ is plotted against the ELM energy, $\mathrm{W}_{\mathrm{ELM}}$, and is in the range of $20-200 \mathrm{eV}$, corresponding to $5-50 \%$ of the ion temperature at the pedestal top. $\mathrm{T}_{\mathrm{i}, \mathrm{ELM}}$ decreases with the separatrix distance and increases with $\mathrm{W}_{\text {ELM. }}$ The former can be explained by the parallel energy loss to the divertor as the filaments propagate radially outwards. The latter suggests that on average the filaments in large ELMs propagate faster radially and have less time to cool due to parallel loss before reaching the far SOL (as was shown in [25], the variation of the initial ELM filament ion temperature - i.e. the filament birth location in the edge plasma - results in small changes of the far SOL $\left.\mathrm{T}_{\mathrm{i}, \mathrm{ELM}}\right)$. This would be consistent with a larger fraction of $\mathrm{W}_{\mathrm{ELM}}$ deposited outside 
the divertor in large ELMs, observed in DIII-D [37] and JET [38, 39]. The ELM filament $\mathrm{v}_{\mathrm{rad}}$ estimated from the measured $j_{\text {sat }}$ and $T_{i, E L M}$ is in the range of 500-2000 m s$~^{-1}$ [25]. The same range of $v_{\text {rad }}$ was previously measured in the AUG far SOL by the FP [2, 40]. Given the dependence of $\mathrm{v}_{\mathrm{rad}}$ on the filament density predicted by some models [27], it is perhaps not surprising that ELM filaments which carry larger $\mathrm{j}_{\text {sat }}$ compared with less intense turbulent filaments are also characterized by somewhat larger $\mathrm{v}_{\text {rad }}$. The same observation was reported from the MAST tokamak [41].

Another piece of evidence that ELMs carry hot ions into the far SOL is obtained from the comparison of the total power flux, $\mathrm{q}_{/ /}$, estimated from the RFA measurements and from an IR camera viewing the probe. An example of such a comparison is shown in figure 10. RFAinferred $\mathrm{q}_{/ /}$is obtained from the measured $\mathrm{j}_{\mathrm{sat}}$ and $\mathrm{T}_{\mathrm{i}, \mathrm{ELM}}=71 \mathrm{eV}$ (for $\mathrm{W}_{\mathrm{ELM}} \approx 28 \mathrm{~kJ}$ ) using the standard sheath-transmission theory [42]. Additionally, we assume the ELM-averaged electron temperature, $\mathrm{T}_{\mathrm{e}, \mathrm{ELM}}=15 \mathrm{eV}$ from earlier LP measurements in AUG [1]. As can be seen from figure 10, RFA and thermographic measurements of $\mathrm{q}_{/ /}$are in a fairly good agreement given the number of uncertainties in a probe-thermography comparison [43] and in calculating $\mathrm{q}_{/ /}$from probe data [44]. Moreover, $\mathrm{T}_{\mathrm{i}, \mathrm{ELM}}$ from figure 9 agrees with earlier estimates of $T_{i, E L M}$ in the AUG SOL, obtained from the first RFA measurements in AUG and from 2-d IR thermography [24, 35].

The RMP in AUG is used for several different probe heads, which places severe restriction on the experimental time dedicated to each probe head. In order to make the RFA measurements in AUG more accessible, a new bi-directional reciprocating RFA, dubbed 'RFA2', has been recently installed inside the AUG torus vessel. RFA2 is located $65 \mathrm{~mm}$ below the outboard midplane and is separated from the RPM toroidally by $180^{\circ}$. As depicted in figure 11, the probe head is mounted on the magnetically-driven reciprocating system identical to that used for the FP in AUG [2]. The torque exerted by the tokamak magnetic field on the energized coil drives the probe into the plasma. Measurements can be currently performed up to about $5 \mathrm{~mm}$ in front of the outboard limiter. Figure 11 shows the signals measured by RFA2 in Type I ELMy H-mode discharges with $\mathrm{W}_{\mathrm{ELM}}=20-30 \mathrm{~kJ}$. The probe sensors were maintained at $\Delta \mathrm{r}_{\text {sep }}=5-5.5 \mathrm{~cm}$ for $400 \mathrm{~ms}$, capturing more than 50 ELMs during the reciprocation. The signals plotted in figure 11 were acquired by the analyzer facing the outboard midplane along $\mathbf{B}$. Also plotted in figure 11 is the current to the inner divertor, $\mathrm{I}_{\mathrm{div}}$, used here as an ELM marker. The collector current measured during ELMs features the same filamentary structure as that observed by the RFA mounted on the RPM (figure 9 and Ref. [25]). Similar to the signals measured by the RFA on the RPM, $I_{c}$ measured during the ELMs decreases with increasing $\mathrm{V}_{\mathrm{g} 1}$. The e-folding voltage is about $30-50 \mathrm{~V}$ for these particular plasma conditions and $\Delta \mathrm{r}_{\text {sep }}$, corresponding to $\mathrm{T}_{\mathrm{i}, \mathrm{ELM}} \approx 30-50 \mathrm{eV}$. This is consistent with $\mathrm{T}_{\mathrm{i}, \mathrm{ELM}}$ estimated earlier from the RFA on the RPM for a given $\Delta \mathrm{r}_{\text {sep }}$ and $\mathrm{W}_{\mathrm{ELM}}$ (figure 9 and Ref. [25]). In addition to the measurements of the far SOL ELM ion energies, RFA2 can be used for the measurements of the rectified sheath potentials in the flux tubes passing in front of the ICRF antenna [45], which is important for reducing deleterious antenna-edge interactions.

\section{The influence of magnetic perturbations on the SOL transport}

The achievement of ELM mitigation is critical in order to avoid damage to in-vessel components in ITER. Studies of the SOL transport in AUG have entered a scarcely explored territory with the newly installed in-vessel magnetic perturbation (MP) coils and the subsequent ELM mitigation achieved [45, 47]. The mitigation was found to be associated e.g. with smaller plasma energy loss and reduced excursions the total divertor power load when type I ELMs were replaced by a stream of smaller, more frequent ELM-like events [45, 47]. Probe measurements at the outboard midplane are to some extend consistent with these observations. As illustrated in figure 12 , in some plasma pulses with MPs, large $\mathrm{j}_{\text {sat }}$ filaments 
associated with type I ELMs are replaced by more continuous and somewhat smaller bursts in mitigated ELMs. However, there are cases where bursts of $j_{\text {sat }}$ remain similar (or become even more pronounced) when type I ELMs undergo transition to mitigated ELMs [48, 49]. Detailed investigation of $j_{\text {sat }}$ dynamics in type $I$ versus mitigated ELMs is a subject of ongoing research. Additionally, figure 9 shows that the far SOL $\mathrm{T}_{\mathrm{i}, \mathrm{ELM}}$ measured in mitigated ELMs with reduced $\mathrm{j}_{\mathrm{sat}}$ excursions is lower compared with $\mathrm{T}_{\mathrm{i}, \mathrm{ELM}}$ in type I ELMs. This observation goes along with the idea that the decrease in the filament density results into more sluggish radial filament advection [27], which makes the filaments cool an a shorter radial distance.

In an L-mode discharge with the plasma density $\mathrm{n}_{\mathrm{e}} / \mathrm{n}_{\mathrm{G}} \approx 0.13$ (with $\mathrm{n}_{\mathrm{G}}$ the Greenwald density), the MPs lead to flattening of $\mathrm{V}_{\mathrm{f}}$ just outside the outboard midplane separatrix and a factor 2 increase of the far SOL $j_{\text {sat }}[48,49]$. The flattening of $V_{f}$ in the SOL is qualitatively consistent with the drop of $\mathrm{E}_{\mathrm{r}}$ in the near SOL during MPs, observed by the Doppler reflectometry $[5,50]$ and shown in figure 13 . The modification of the near SOL $E_{\mathrm{r}}$ during MP is significant at low $\mathrm{n}_{\mathrm{e}} / \mathrm{n}_{\mathrm{G}}$, but is reduced with increasing the plasma density. Above $\mathrm{n}_{\mathrm{e}} / \mathrm{n}_{\mathrm{G}} \approx$ 0.3 the effect of MPs on the near SOL $\mathrm{E}_{\mathrm{r}}$ is negligible. The statistical moments and the gradient of $\mathrm{j}_{\text {sat }}$ measured by the LPs in the SOL are not affected by MPs. At $\mathrm{n}_{\mathrm{e}} / \mathrm{n}_{\mathrm{G}} \approx 0.25$ the LPs see little, if any, effect of MPs on the SOL, which is consistent with the reflectometric observations.

Further effect of MPs on the SOL transport near the X-point observed in AUG is illustrated in figure 14. The inset panel shows an R-z plot of the minimum normalized flux surface that each field line experiences during its trajectory, $\Psi_{\min }=\min \left[\left(\Psi-\Psi_{\text {axis }}\right) /\left(\Psi_{\text {sep }}-\right.\right.$ $\Psi_{\text {axis }}$ )] (with $\Psi_{\text {axis }}$ and $\Psi_{\text {sep }}$ being respectively the poloidal fluxes at the magnetic axis and at the separatrix). $\Psi_{\min }$ is obtained from vacuum perturbation fields and translates into a relative depth of the field line penetration into the plasma, which largely determines the heat flux arriving to the divertor targets. Finger-like offshoots due to MPs, first introduced in Ref. [51] and recently observed experimentally on MAST [52], are clearly seen near the X-point. Some of the lobes intersect the divertor target and channel relatively large power flux to the divertor, which leads to several maxima in divertor power load profile observed in figure 14 . It is worth highlighting that the measured splitting of the divertor strike zones, observed also in L-mode at low plasma density (figure 15 and Ref. [49]), is consistent with the prediction from the EMC3-Eirene simulation of the same discharge [53] (radiated power to the divertor, neglected in the simulation, can explain a factor 2 larger divertor power load from IR measurements in figure 14). The agreement also indicates that at least at low plasma density the lobe structure can be described accurately enough by the vacuum approach as in the EMC3-Eirene.

\section{Summary}

Latest measurements in the AUG SOL summarized in this contribution bring a number of new important information on turbulence and transport in the tokamak plasma boundary.

A relation between the plasma and floating potential fluctuations has been studied experimentally and by gyrofluid simulations. The results indicate that the 'standard' assumption $\tilde{V}_{\mathrm{p}} \approx \tilde{\mathrm{V}}_{\mathrm{f}}$ is physically invalid due to electron temperature fluctuations and can yield erroneous measurements of the turbulence-induced particle flux. These studies have emphasized the importance and feasibility of direct plasma potential measurements.

New measurements by an RFA in AUG provide further evidence that both, ELM and turbulent filaments carry hot ions over large radial distances in the SOL. The measured far SOL ELM ion temperature is up to $50 \%$ of the pedestal top temperature and increases with the ELM energy. This observation can be explained by faster radial advection of filaments in large ELMs and is consistent with earlier results from JET and DIII-D where large ELMs were found to deposit larger fraction of their energy outside the divertor. Moreover, RFA 
experiments in AUG have also demonstrated that an RFA can provide valuable data from harsh $\mathrm{H}$-mode conditions and can motivate further use of this technique across devices.

Studies of the SOL transport in AUG have acquired a greater significance with the newly installed in-vessel magnetic perturbation (MP) coils and the subsequent ELM mitigation achieved. Large ion current filaments seen in the SOL in type I ELMs are replaced by more continuous and somewhat smaller bursts in mitigated ELMs produced by in-vessel magnetic perturbation coils. Additionally, RFA measurements suggest the ions in the far SOL in mitigated ELMs are colder compared with those measured in type I ELMs at the same separatrix distance. Observing that extreme transport events in the SOL fade away as type I ELMs undergo transition to mitigated ELMs goes along with earlier findings in AUG that e.g. plasma energy loss and divertor power load drop after the transition to mitigated ELMs. Finally, the splitting of the divertor strike zones observed by the infrared imaging in H-mode with MPs has been compared with the prediction from the EMC3-Eirene simulations. A reasonable agreement between the thermographic measurements and the simulations suggest that 'lobe' structures due to perturbation fields observed near the X-point are not significantly affected by plasma screening, and can be described by vacuum perturbation fields.

\section{Acknowledgements}

This work was supported in part by the Austrian Science Fund (FWF) Y398, projects MSMT LG11018 and P205/12/2327 of the Czech Science Foundation, and by the GA CR grant No. GA202/09/1467.

\section{References}

[1] H.W. Müller et al., Nucl. Fusion 51 (2011) 073023.

[2] A. Schmid et al., Plasma Phys. Control. Fusion 50 (2008) 045007.

[3] M. Weinlich et al., Contrib. Plasma Phys. 36 (1996) 53.

[4] M. Tsalas et al., J. Nucl. Mater 363-365 (2007) 1093.

[5] G.D. Conway et al., Plasma Phys Control. Fusion 46 (2004) 951.

[6] R.A. Pitts et al., J. Nucl. Mater. 415 (2011) S957.

[7] B. Nold et al., New Journal of Physics 14 (2012) 063002.

[8] F.P. Gennrich et al., Plasma Phys. Control. Fusion 54 (2012) 015012.

[9] B.D. Scott, Phys. Plasmas 12 (2005) 102307.

[10] J. Adámek et al., Contrib. Plasma Phys. 48 (2008) 395.

[11] J. Adámek et al., J. Nucl. Mater. 390-391 (2009) 1114.

[12] D.A. D'Ippolito, Phys. Plasmas 18 (2011) 060501.

[13] M. Kočan et al., J. Nucl. Mater 415 (2011) S1133.

[14] N. Bisai, R. Singh and P.K. Kaw, Phys. Plasma 19 (2012) 052509.

[15] J.R. Angus and S.I. Krasheninnikov, Phys. Plasmas 19 (2012) 052504.

[16] I. Furno et al., Phys. Plasmas 15 (2008) 055903.

[17] K. Okazaki, H. Tanaka, N. Ohno, N. Ezumi Y. Tsuji and S. Kajita, Rev. Sci. Instrum 83 (2012) 023502.

[18] B. Nold et al., Plasma Phys. Control. Fusion 52 (2010) 065005.

[19] M. Kočan et al., Plasma Phys. Control. Fusion 54 (2012) 085009.

[20] F.P. Gennrich et al., 'Numerical investigation on the validity of ion temperature measurements by a retarding field analyzer in turbulent plasma', Proc. $39^{\text {th }}$ EPS Conf. on Plasma Phys. (Stockholm, 2012) P2.082 http://ocs.ciemat.es/epsicpp2012pap/pdf/P2.082.pdf

[21] W. Fundamenski et al., Plasma Phys. Control. Fusion 48 (2006) 109.

[22] R.A. Pitts et al., Nucl. Fusion 46 (2006) 82.

[23] R.A. Pitts et al., J. Nucl. Mater. 390-391 (2009) 755. 
[24] M. Kočan et al., Plasma Phys. Control. Fusion 53 (2011) 065002.

[25] M. Kočan et al., Nucl. Fusion 52 (2012) 023016.

[26] A. Kirk et al., J. Nucl. Mater. 390-391 (2009) 727.

[27] O. E. Garcia, H. H. Bian, W. Fundamenski, Phys. Plasmas 13 (2006) 082309.

[28] M. Kočan et al., Contrib. Plasma Phys. 53 (2013) 22.

[29] A.S. Wan, T.F. Yang, B. Lipschultz and B. LaBombard, Rev. Sci. Instrum. 57 (1986) 1542 .

[30] I. Katsumata, Contrib. Plasma Phys. 36 (1996) S 73.

[31] J. Adámek et al., 'Fast ion temperature measurements using ball-pen probes in the SOL of ASDEX Upgrade during L-mode', Proc. $38^{\text {th }}$ EPS Conf. on Plasma Phys. (Strasbourg, 2011) P1.059 http://ocs.ciemat.es/EPS2011PAP/pdf/P1.059.pdf

[32] G.F. Matthews, J. Phys. D: Appl. Phys. 17 (1984) 2243.

[33] M. Kočan et al., J. Nucl. Mater. (2013), http://dx.doi.org/10.1016/j.jnucmat.2013.01.103 (in press).

[34] A. Herrmann et al., Plasma Phys. Control Fusion 46 (2004) 971.

[35] A. Herrmann et al., J. Nucl. Mater. 363-365 (2007) 528.

[36] P. Tamain, M. Kočan, J.P. Gunn, A. Kirk, J.-Y. Pascal and M. Price, J. Nucl. Mater. 415 (2011) S1139.

[37] Loarte A. et al 2006 Proc. 21st Int. Conf. on Fusion Energy 2006 (Chengdu, China, 2006) (Vienna: IAEA) CD-ROM file IT/P1-14 and

http://www-naweb.iaea.org/napc/physics/FEC/FEC2006/html/index.htm

[38] T. Eich et al., J. Nucl. Mater. 337-339 (2005) 669.

[39] W. Fundamenski et al., Nucl. Fusion 51 (2011) 083028.

[40] A. Kirk et al., Plasma Phys. Control. Fusion 53 (2011) 035003.

[41] N. Ben Ayed et al., Plasma Phys. Control. Fusion 51 (2009) 035016.

[42] P.C. Stangeby, Phys. Fluids 27 (1984) 682.

[43] J. Marki et al., J. Nucl. Mater. 363-365 (2007) 382.

[44] J.P. Gunn et al., J. Nucl. Mater. (2013), http://dx.doi.org/10.1016/j.jnucmat.2013.01.055 (in press)

[45] V. Bobkov et al., 'ICRF operation with improved antennas in a full W-wall ASDEX Upgrade, status and developments', submitted to Nuclear Fusion (January 2013).

[46] W. Suttrop et al., Plasma Phys. Control. Fusion 53 (2011) 124014.

[47] W. Suttrop et al., in Fusion Energy 2012 (Proc. 24th Int. Conf. San Diego, 2012) (San Diego, USA 8 - 13 October 2012)

http://www-naweb.iaea.org/napc/physics/FEC/FEC2012/index.htm

[48] H.W. Müller et al., 'Effect of Non-axisymmetric Magnetic Perturbations on the Turbulence at Open Field Lines in ASDEX Upgrade',Proc. $38^{\text {th }}$ EPS Conf. on Plasma Phys. (Strasbourg, 2011) P4.122. http://ocs.ciemat.es/EPS2011PAP/pdf/P4.122.pdf

[49] H.W. Müller et al., J. Nucl. Mater. (2013), http://dx.doi.org/10.1016/j.jnucmat.2013.01.010 (in press)

[50] G.D. Conway et al., Plasma Fusion Res. 5 (2010) S2005.

[51] T.E. Evans et al., Contrib. Plasma Phys. 44 (2004) 235.

[52] A. Kirk et al., Phys. Rev. Lett. 108 (2012) 255003.

[53] T. Lunt et al., Nucl. Fusion 52 (2012) 054013. 


\section{Tables}

Table 1. Selected probes heads used on the RPM featured in this paper and their primary use. The probe heads are listed as they appear in the paper.

\begin{tabular}{ll}
\hline Probe head & Primary use \\
\hline Turbulence probe & Fluctuations of electron temperature and density \\
Ball pen probe (BPP) & Plasma potential and ion temperature \\
Retarding field analyzer (RFA) & Ion energies in ELMs and turbulent filaments, \\
$\mathbf{E} \times \mathbf{B}$ analyzer & Fast ion temperature measurements \\
\hline
\end{tabular}


Figures

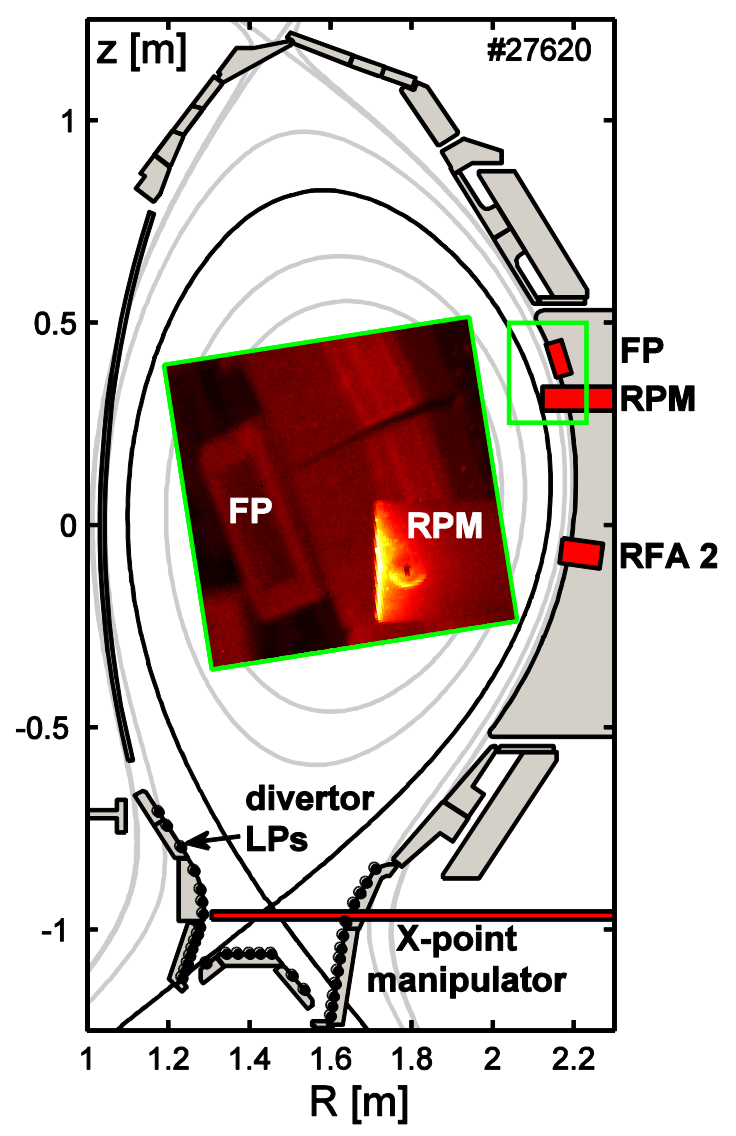

Figure 1. Poloidal cross section of AUG, showing a typical lower single-null plasma equilibrium and the location of the reciprocating probes and the divertor Langmuir probes. Inset panel illustrates the infrared camera view. 

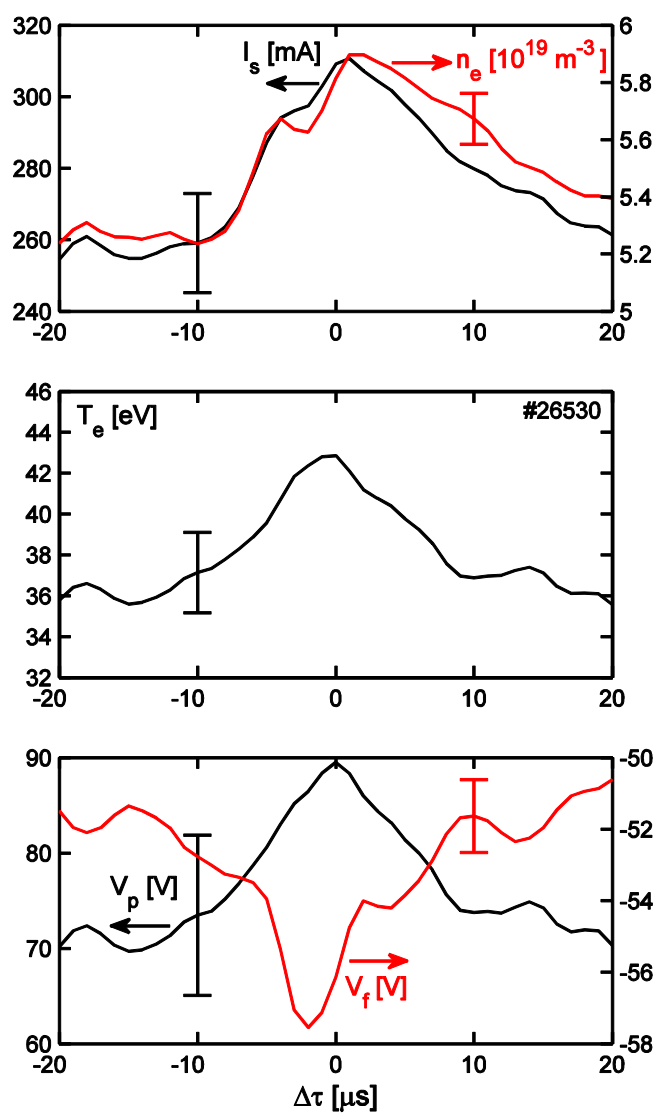

Figure 2. From top to bottom: fluctuations of ion saturation current, electron density, electron temperature, plasma potential and floating potential measured from conditionallysampled I-V characteristics. Also shown are representative error bars. 


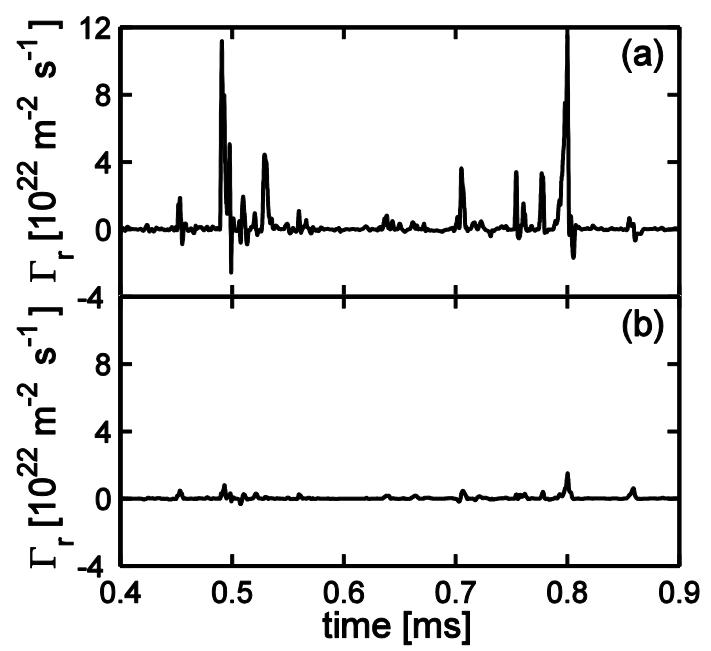

Figure 3. Radial particle flux density in the SOL derived from (a) synthetic probe measurements of ion current and floating potential fluctuations and (b) obtained directly from simulated $\tilde{n}_{e}$ and $\tilde{V}_{p}$. 


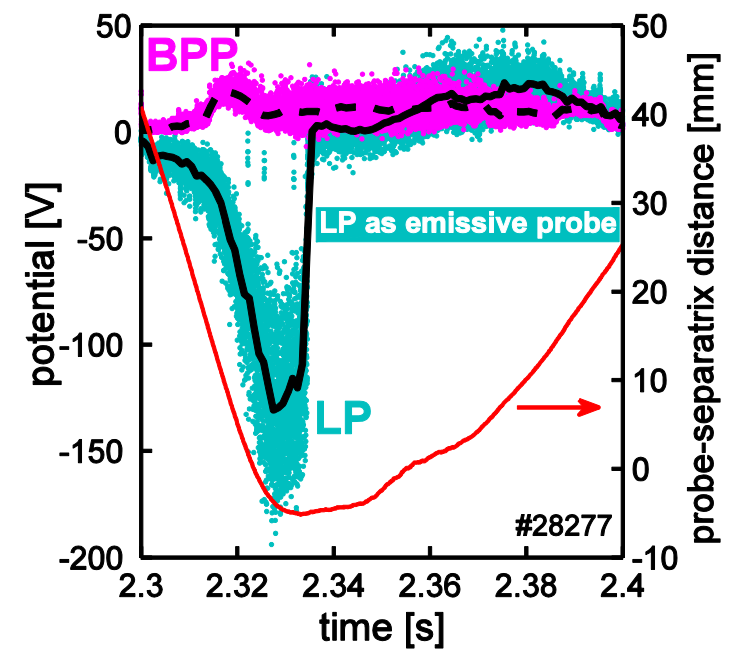

Figure 4. Potentials measured by a ball-pen probe and an electrically floating Langmuir probe. At $t \approx 2.335 \mathrm{~s}$ the LP undergoes a transition to an emissive probe and measures the plasma potential together with the BPP. 

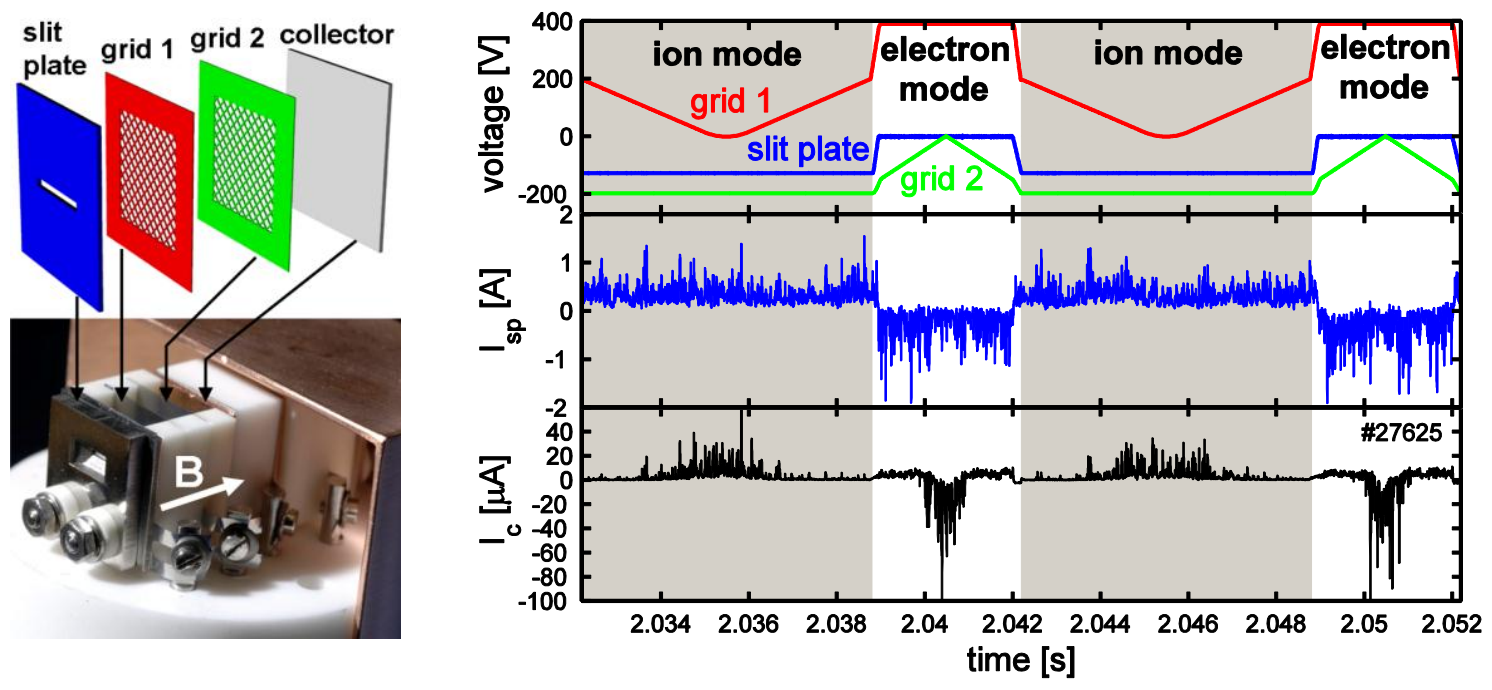

Figure 5. Left: Photograph and schematic of the RFA. Right: time traces of the bias voltages applied to RFA electrodes in the 'ion' and the 'electron' mode and the corresponding currents measured at $\Delta r_{s e p}=2.5 \mathrm{~cm}$ by the RFA slit plate $\left(I_{s p}\right)$ and the collector $\left(I_{c}\right)$ facing the outboard midplane along $\boldsymbol{B}$. 


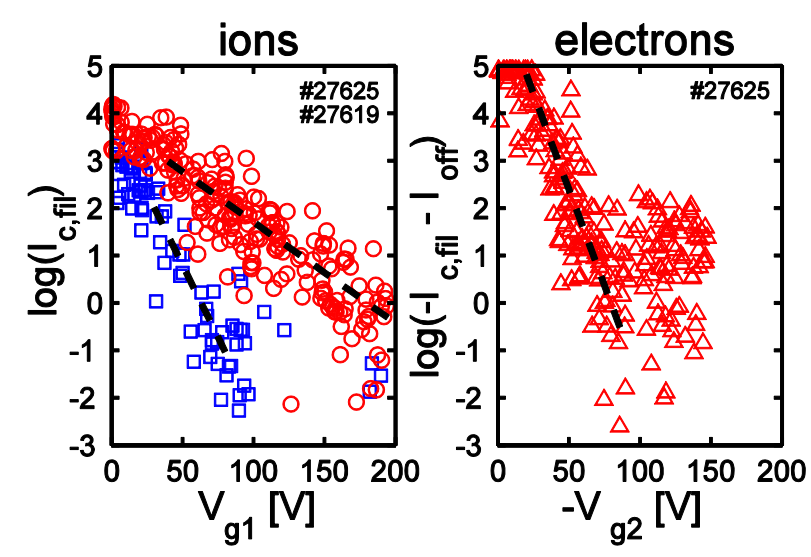

Figure 6. Conditionally-sampled filament ion (left) and electron (right) I-V characteristics measured by the RFA in ohmic discharges. Filaments characterized by the peak slit plate current (2.5-3.5) $\sigma$ above the time averaged mean are included. Dashed: exponential fit to the decaying part of the ion I-V characteristic which yields $T_{i, f i l} \approx 47 \mathrm{eV}$ (circles) and $T_{e, f i l} \approx 12$ $\mathrm{eV}$ (triangles) at $\Delta r_{\text {sep }}=25 \mathrm{~mm}$ and $T_{i, f i l} \approx 16 \mathrm{eV}$ (squares) at $\Delta r_{\text {sep }}=45 \mathrm{~mm}$. Note that the collector current signal saturates at $\log \left(-I_{c, f i l} I_{o f f}\right)=4.9$. 


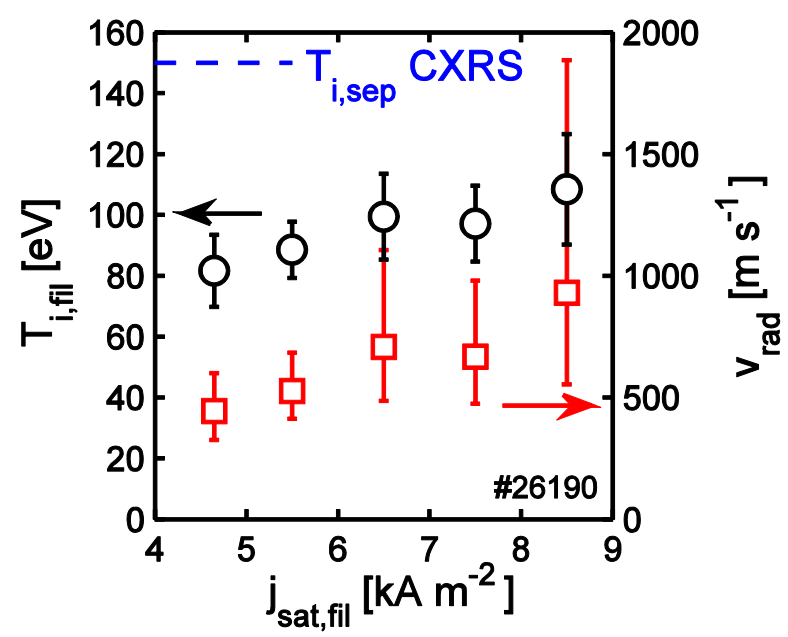

Figure 7. The filament ion temperature (circles) measured at $\Delta r_{\text {sep }}=21 \mathrm{~mm}$, plotted against the filament ion current density. The values of $j_{\text {sat,fil }}$ correspond to (1-4.5) $\sigma$ above the time averaged mean. Squares denote the radial filament propagation speed required in the parallel loss model to match $T_{i, f i l}$. 


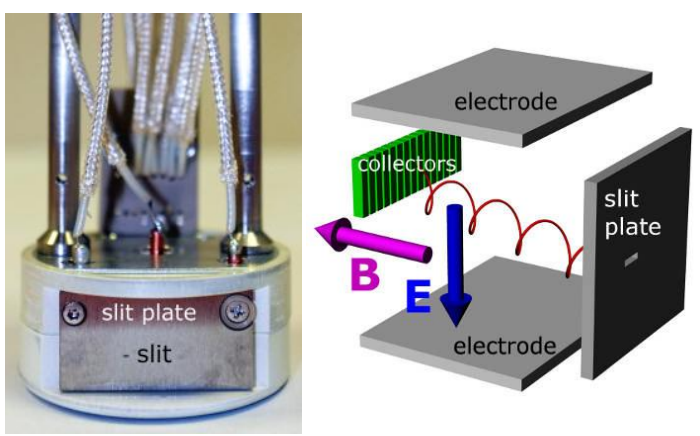

Figure 8. $\boldsymbol{E} \times \boldsymbol{B}$ analyzer without the protective graphite housing (left) and the schematic of the analyzer (right). 


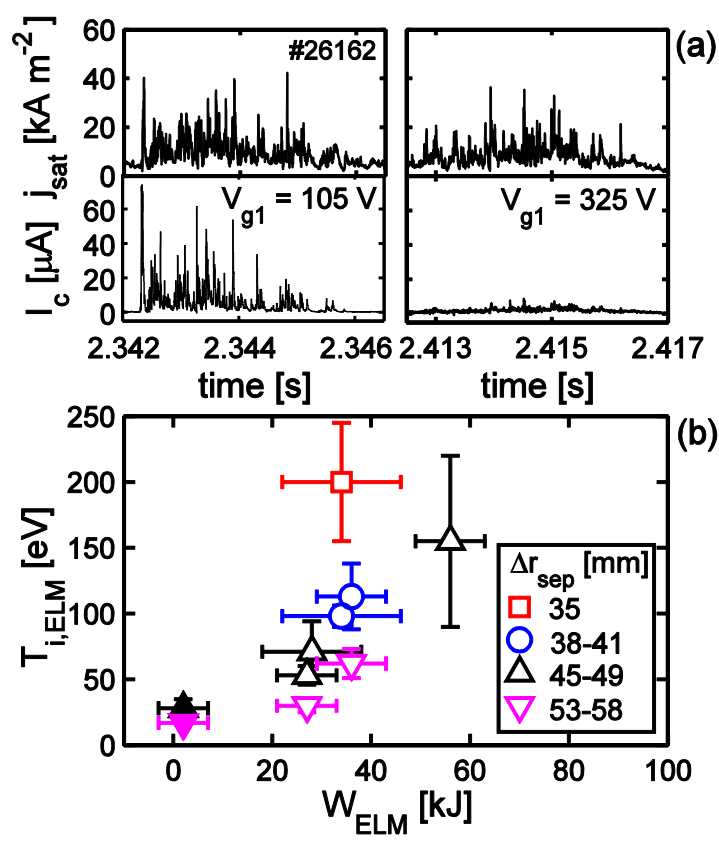

Figure 9. (a) Ion current density and the collector current measured by the $R F A$ at $\Delta r_{\text {sep }}=35$ mm in two similar type I ELMs. (b) Far SOL ELM ion temperature plotted against the ELM energy. Filled symbols: mitigated ELMs. 

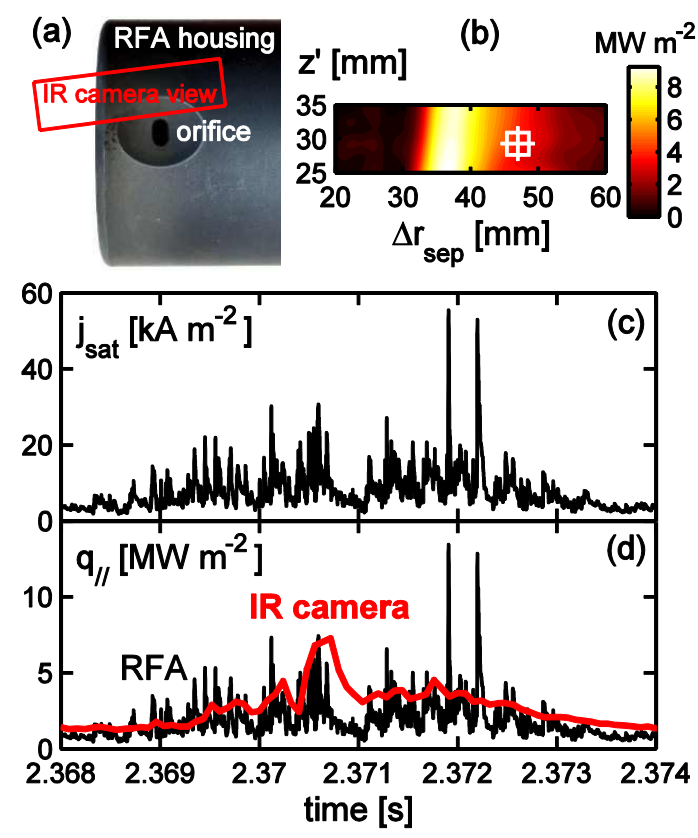

Figure 10. (a) IR camera view of the RFA. (b) IR snapshot taken during type I ELM. (c) ELM ion current density and (d) RFA-inferred power flux at $\Delta r_{\text {sep }}=47 \mathrm{~mm}$. Also plotted is q// from the IR camera with the ROI at the same $\Delta r_{\text {sep }}$ as the RFA sensors (\#26164). 

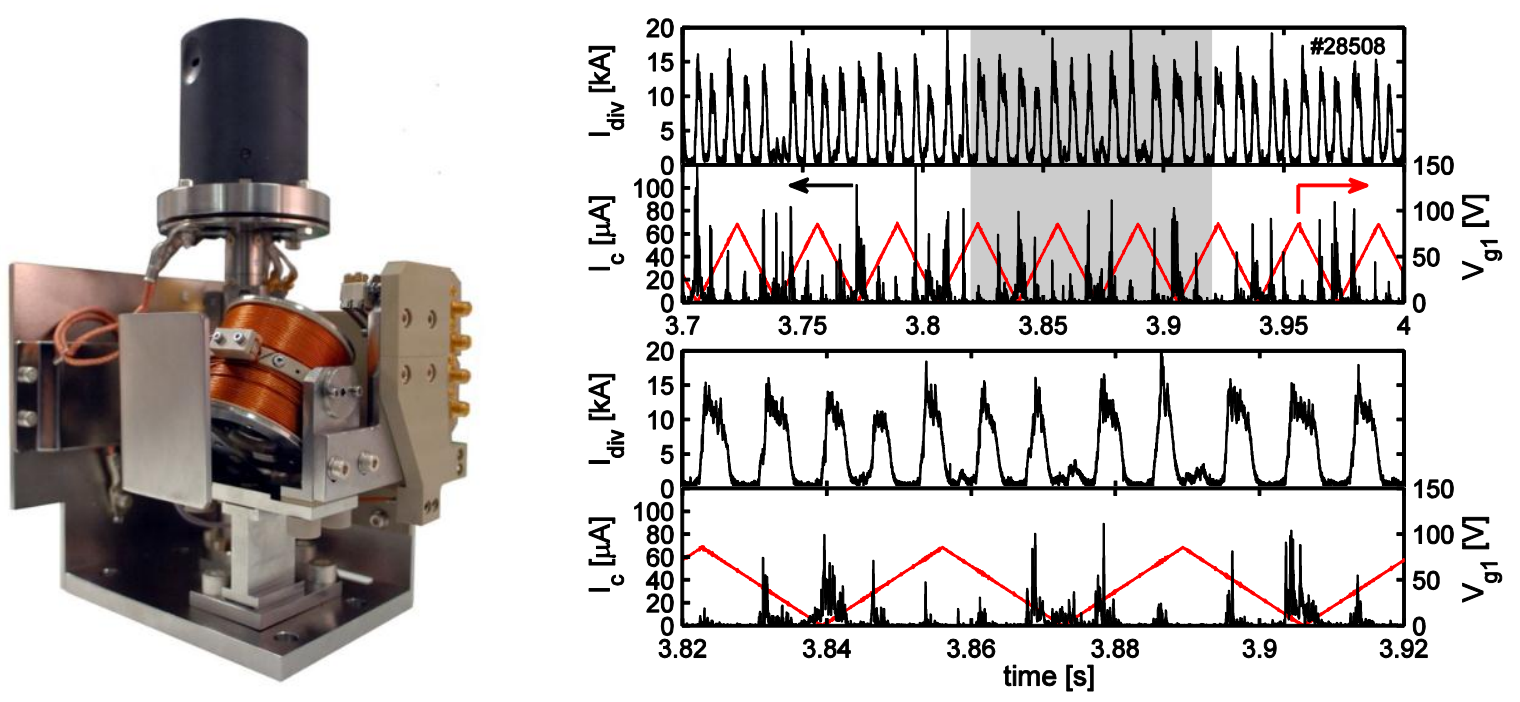

Figure 11. Left: Magnetically-driven reciprocating RFA2 before the installation inside the AUG torus vessel. Right: Time traces of the inner divertor shunt current, the bias voltage applied to grid 1, and the collector current measured in the far SOL by the analyzer facing the outboard midplane. Signals were measured in the Type I ELMy H-mode discharge. The RFA slit plate and grid 2 were biased negatively. Time traces measured for $t=3.82-3.92 \mathrm{~s}$ are expanded. 

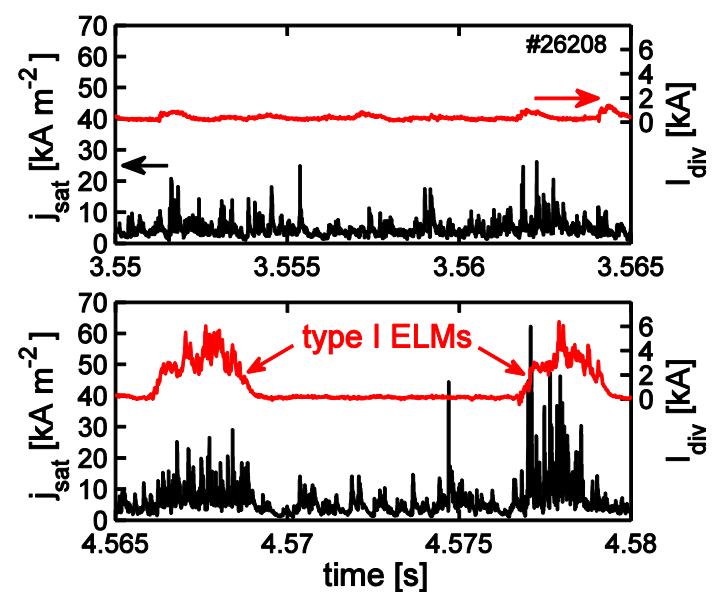

Figure 12. Ion saturation current density at $\Delta r_{\text {sep }}=47 \mathrm{~mm}$ and the inner divertor shunt current measured in mitigated (top) and type I ELMs (bottom) in otherwise similar conditions. 


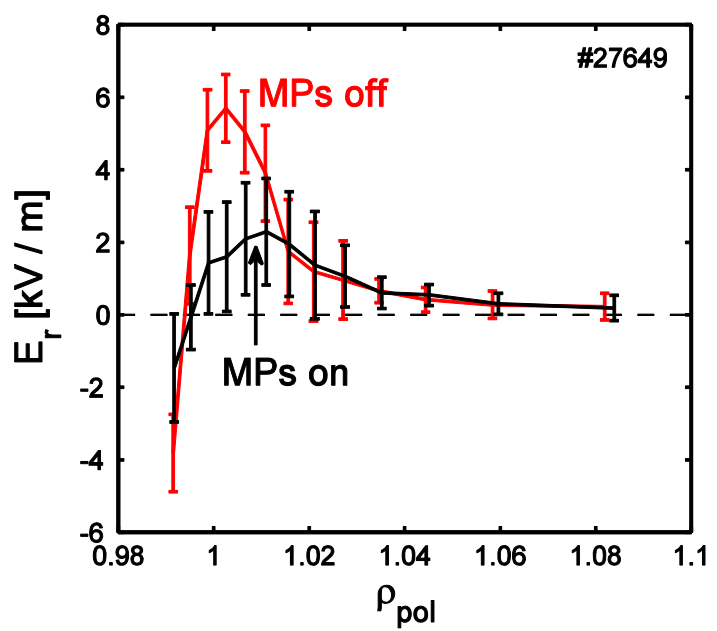

Figure 13. The effect of MPs on $E_{r}$ near the separatrix measured by the Doppler reflectometry in the L-mode discharge characterized by $B_{t}=-2.5 T, I_{p}=1.0 M A, q_{95} \approx 4.2$, $n_{e} / n_{G} \approx 0.23$, and $n=2$ odd parity MPs. 


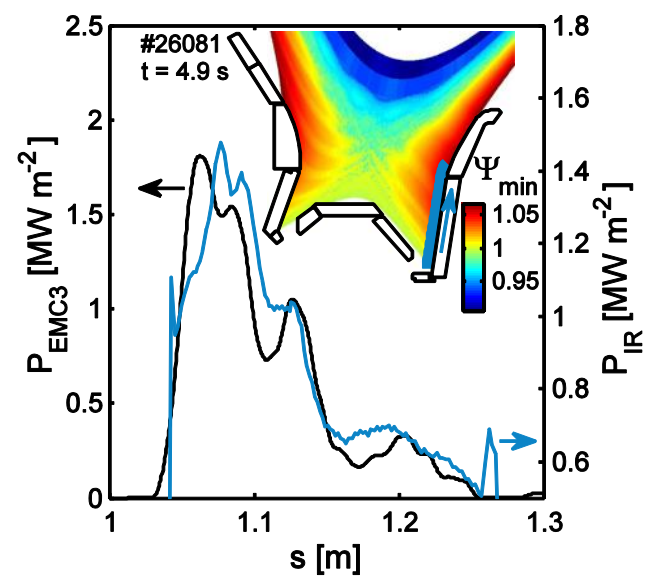

Figure 14. Outer divertor power flux density in H-mode discharge with magnetic perturbations. EMC3 Eirene simulation is compared with the divertor thermography. Inset shows a poloidal map of $\Psi_{\min }($ see text). Thick line corresponds to $s=1-1.3 \mathrm{~m}$. 


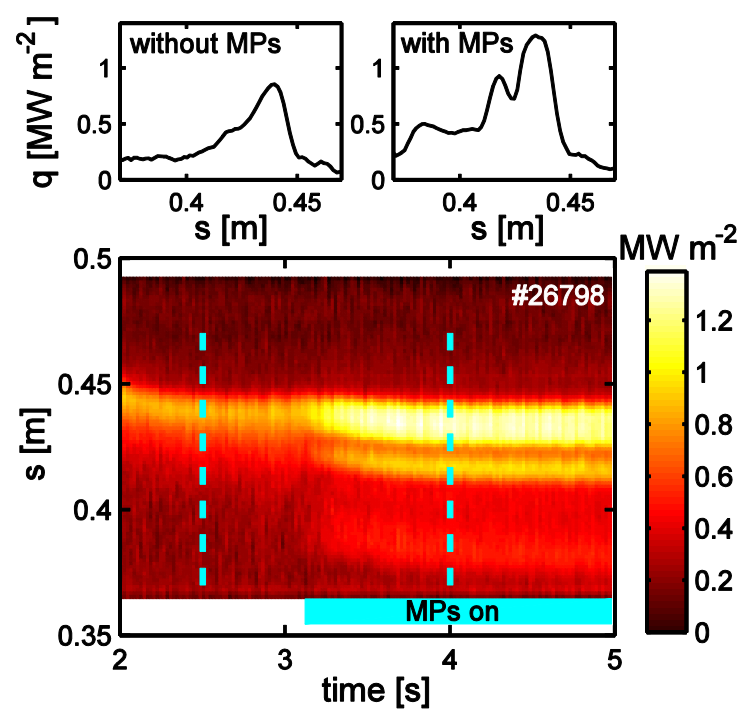

Figure 15. Time evolution of the IR-inferred heat flux density to the inner divertor, illustrating the splitting of the divertor strike zones due to MPs in the L-mode discharge with $n=2$ in odd parity MPs. Snapshots above were taken at the times indicted by the dashed lines. 